\title{
Aspects of Methodology for Interaction Analysis
}

\author{
Carl Vogel \\ Computational Linguistics Group \\ Trinity College Dublin \\ The University of Dublin \\ Ireland \\ Email: vogel@scss.tcd.ie
}

\author{
Maria Koutsombogera \\ Computational Linguistics Group \\ Trinity College Dublin \\ The University of Dublin \\ Ireland \\ Email: koutsomm@scss.tcd.ie
}

\author{
Anna Esposito \\ Università degli Studi della Campania \\ "Luigi Vanvitelli" \\ Caserta, Italy \\ and \\ International Institute for \\ Advanced Scientific Studies (IIASS) \\ Vietri sul Mare, Italy \\ Email: iiass.annaesp@tin.it
}

\begin{abstract}
Observational methods in interaction analysis are defended. Observational methods are distinguished from experimental methods with respect to the mode of data collection. Other approaches to human interaction analysis considered are: prospective, technological, psychoanalytic and philosophical.
\end{abstract}

\section{INTRODUCTION}

Advances in technologies associated with artificial intelligence and cognitive infocommunications are frequently underpinned by advances in understanding of human behaviours. Recent works have attempted to characterize the scope of interaction analysis [1], [2]. Given the scope of the cognitive infocommunications field and its relationships to cognate disciplines from artificial intelligence to zoology, it is natural that a plethora of methodological approaches are evident in works that address interaction analysis under the aegis of cognitive infocommunications. Nonetheless, we find it helpful to explicitly defend an observational approach, and that is the purpose of this paper.

We view of interaction analysis as the study of how humans interact with themselves, each other and non-humans, using language, gesture (including laughter) and physical placement. Interaction, in this sense, is an ancient discipline [3], [4]. The rise of artificial interaction agents and mobile communication devices have invigorated explorations in this area, typically in the hope of developing devices that display interaction behaviours that are like those of humans. The pursuit of artificial agents as new technologies emphasizes engineering oriented methods that scale towards "big data". However, there remains a role for observational methods attached to "small data" [5], [6], as has been defended within linguistics in the past [7], and data sets in between, such as given by natural language corpora, including multi-modal corpora [8], [9].

This paper is structured as follows. Firstly, ( $(I I)$ we elaborate on what we take to be the content of interaction analysis. Then we explain what we take to be constitutive of an observational method as a paradigm for research in interaction analysis ( (III). We defend this approach as a properly scientific method ( $\mathrm{IV}$. We then observe the range of methodological approaches adopted within interaction analysis, using a small but representative sample $(\$ \mathrm{~V})$. Finally, we conclude that researchers need not in each work defend the choice of an observational per se, but rather, with respect to the research questions they pursue (\$VI).

\section{INTERACTION AS AN OBJECT OF STUDY}

In contrast to sentence level grammar, where syntactic rules and constraints appear to be explanatory, it is less obvious that instances of interaction follow rules of well-formedness. The main evidence that there might be follows from diagnosis of schizophrenia attending to disordered interaction patterns [10]-[13]. Interaction grammars appear to be implicit in foundational work on artificial dialogue systems. In general, though, there appear to be as many rules as the interaction contexts, the speakers' intentions in them, the composition of the interaction groups etc. Thus, observational studies are necessary also because of the variability of interaction instances. Consider the turn-taking system and its rules, functioning as a descriptive framework for dialogue. Instantiations of turntaking though can be numerous, especially when considering multi-modal ways of doing so - and they most probably serve communication goals. In general, aberrations from the turntaking rules, e.g. overlaps, should not be interpreted similarly to ungrammatical word or sentences, because they tend to have a function in the dialogue.

There is inherent interest in understanding how humans interact in gatherings of varying scale, just as there is inherent interest in understanding planetary motions and subatomic particles. However, there is also increasing demand for technology that replicates human interactions, and the challenges associated with such technologies includes the need to understand human interactions at varying levels and across contexts [14].

In the behavioral analysis domain, a common way to have a controlled methodology of a research topic and the data accompanying it are academic challenges and competitions such as the Interspeech Computational Paralinguistics ChallengE (ComParE) 11 Emotion Recognition in the Wild (EmotiW) ${ }^{2}$ AudioVisual Emotion Challenge (AVEC) ${ }^{3}$ and ChaLEARN Looking At People (LAP) 4 Organizers of those challenges formulate the problem, provide data and evaluation metrics, as well as forums for the dissemination of results, aiming to advance the state-of-the-art in that particular topic.

\footnotetext{
${ }^{1}$ http://www.compare.openaudio.eu// last accessed 26.06.2020

${ }^{2}$ https://sites.google.com/view/emotiw2020 last accessed 26.06.2020

3 https://sites.google.com/view/avec2019/home last accessed 26.06.2020

4 http://chalearnlap.cvc.uab.es/ last accessed 26.06.2020

${ }^{5}$ Links to the above challenges point to the website of the latest challenge organised, and include an inventory of past challenges.
} 
Consider the example of personality computing in all three dimensions of automatic prediction, perception and synthesis of personality traits [15]-[17]. Personality is a topic already well-defined theoretically, as it is based on extensively tested psychological theories. Therefore, the computing aspect is related to engineering approaches aiming to create models that best predict the personality traits (e.g. [18]). As constructed within the "big-five" inventory of traits, traits are relational in the sense that scores in any one dimension encode an individual's percentile position - the percentage of people who have the trait to a lesser extent. Primary data in this area includes the scores that are appropriate to individuals' responses to validated instruments, and these amount to individuals' observations of their own preferences and their behaviours in interactions. Thus, the primary data of interaction analysis may include that which follows results of individuals' introspection, direct interaction with others, or indirect, relational accounts.

\section{A PARADIGM FOR ANALYZING INTERACTION}

With reference to natural languages, it is typical to think of the inflection patterns for words as paradigms [19]: given a verb (e.g. forsake) and the information that it has the same conjugation pattern as another verb (e.g. take), one can reach a reliable conclusion about how to form its past perfect (e.g. forsaken). This notion of paradigm is one of the senses of the term paradigm as used in the philosophy of science [20], [21]. Given a particular method, one may apply that method to a range of cognate questions in search of partial answers.

Here we describe a paradigm for interaction analysis anchored in the scrutiny of multi-modal corpora [22]. Observational is an apt single-word characterization. Observation is always conducted within a theoretical framework that provides an inventory of qualities which are relevant to quantify [23]. Observations are particulars, and particulars form the basis of generalizations, hypotheses that may be put to additional testing, towards refining the theoretical framework. Experimental studies are devised in order to test hypotheses about effects involving the qualities observed. An enormous methodological literature provides conventions for determining whether one may reject the null hypothesis that the effects are random interactions, accepting the alternative hypothesis that the qualities involved have explanatory force. Those experimental methods are useful within observational studies as well, in assessing whether the qualities noticed interact with each other in interesting ways. Further, data that informs an observational study in one endeavour may inform an experimental study in another, whether the contrasting conditions are available wholly within the data or the data is combined with other data sets in order to establish the relevant contrasts and controls.

Any number of multi-modal corpora exist. The Switchboard corpus includes speech and text modalities [24], [25]. The HCRC Map Task corpus involves a collaborative task that may be achieved only through linguistic interaction, and is recorded in speech and text [26] ${ }^{6}$ HuComTech involves simulated interviews and structured informal interactions, recorded in video, speech and text [27], [28]. The ILMT-s2s involves a map-task construction but places machine translation between speakers of English and Brazilian Portuguese, recording video,

${ }^{6}$ Video was also recorded, but is not available in the corpus releases. audio, text, eye movements and bio-signals [29]. The MULTISIMO data set includes video, audio and text of three party interactions in a collaborative task [22]. The MULTISIMO corpus was designed to make available an English corpus that would support the analysis of qualities of interaction (gaze, gesture, linguistic content) in relation to participant qualities (personality traits, as measured using the big-five personality inventory) and perception by external parties (of participant dominance and interlocutor collaboration).

Consider dominance [30]. Five independent evaluators rated participants in each of the conversations of the MULTISIMO corpus, using a five point scale, and a definition of dominance as "a person's tendency to control the behaviour of others when interacting with them". The question included whether dominance perceived by independent observers would relate directly to combinations of personality trait scores that arose from the dialogue participants own responses to a personality inventory and whether dominance would relate to the balance of contributions from participants to the dialogues. At face value, "the balance of contributions" is a straightforward notion. However, it may be made operational in any number of ways: in relation to the total duration of speaking, the total number of words or turns, the total number of words uttered relativized to speaking time, the counts of interruptions leading to "owning" the floor, and so on. In analyzing the MULTISIMO data, it emerged that relations of both sorts were visible (highest levels of extroversion linked to high dominance, and of agreeableness to low dominance; greater rate of speech in words per minute linked to higher dominance) $[30]$. However, this is not the end of the scrutiny of either personality traits and dominance or conversational behaviours and dominance within this data. It must still be considered whether that work made operational the main constructs in the most sensible manner. For example, the dominance scores of the five judges were aggregated as medians rather than means. Another alternative is to analyze disagreement about dominance ratings as a primary variable, and then, for example, a greater number of words spoken per minute corresponds to greater disagreement among the dominance judges [31].

Concepts we study-collaboration, dominance, personality, etc.-are not directly measurable from the raw data, hence our approach to construct new ways of making those concepts operational and test the quantitative interactions among those qualities. In the literature, directly measurable features from voice, turn-taking, gestures etc. are often mentioned as "lowlevel" and dominance and the like as "high level". We are not aware of any particular theoretical grounding of high level features in that related literature. Indeed, much empirical work using openSMILE [32] attempts to understand how "low level" features cluster and inform "high-level" labeling judgements. The 'truth' is normally about human assessments serving as a test-bed for the approaches employed each time.

The approach to the data that we are engaged in is a series of exercises in which we explore the data on the basis of theoretical perspectives (e.g. the discourse function of laughter, the signs of collaboration, the role of gaze). The sum of this series is a deeper insight into the particulars of the MULTISIMO corpus as well as the generalizations that hold for other collaborative corpor, and beyond. Thus, the paradigm of exploration involves making operational theoretical constructs in terms 
of the data at hand, and then measuring relationships among those operationalized qualities. We explore whether these same relationships hold for alternative operationalizations and we use the relationships that hold to inform our general theories. For example, one may have a general theory of how greater or lesser collaboration may be manifest in a collaborative corpus; such a general theory is elaborated by qualifying it with a regression equation that fits the data to whatever degree of goodness for the MULTISIMO data set, and which may thereafter tested on alternative data sets (to the extent that those data sets support the same operationalizations).

\section{DEFENDING THE PARADIGM}

One might wonder whether it is really necessary to defend the paradigm that we have described. Our experience has been that it is necessary to defend the approach against a number of critiques, such as that research which uses the observational approach is not guided by a coherent theoretical framework or that it is a "statistical significance fishing expedition".

As suggested above, theory-free observations are impossible. Theoretical qualities that may be explored can be made operational in countless ways, each of them anchored in wellreasoned rationality. Observational studies allow one to obtain information about useful operationalizations of theoretical constructs. Some of those operationalized notions participate in effects that are statistically significant and some do not. If one applies Bonferroni correction as a filter, then one can feel more confident that the effects identified as significant would also emerge in an experimental study of the same effect, and that one is not in a situation in which an estimated probability less than 0.05 is an instance of an event better explained by chance than the hypothetical effect. However, in some cases, visible effects are not even statistically significant: this does entail that they are not significant in a non-statistical sense ${ }^{7}$ Tests of interactions among effects from inferential statistics have a role in observational studies as well as in experimental studies. Their use in observational studies does not amount to "statistical significance fishing", but rather to "significance fishing" in the wider sense of identifying patterns in the data that signify processes and relationships underlying the data.

Where one has a theory that one is extremely confident to be true, one might imagine that there would be little reason to engage in demonstrations of the truth of the theory. Instead, what might appear to be attempts to demonstrate truth are actually occasions for potential falsification. The disproof of a theory is as important to theory building as the discovery of generalizations. The identification of generalizations evident in phenomena is at the heart of theory construction, and is the methodological goal of observational approaches. The same data that supports identification of generalizations may also be useful in realizing constraints that apply to the generalizations. However, in articulating theories that arise in this fashion one is aware that, as theories, they need to be tested with additional data and in experimental conditions.

A potential critique of observational studies is that they are premature. Given the difference in the standard of knowledge that arises from a controlled experimental study and an observational study, and given the extreme volume of studies put

\footnotetext{
${ }^{7}$ Recall that a type II error is the failure to reject a false null hypothesis.
}

to peer review in the face of limited publication space, there is a natural inclination to prefer research that has a controlled experimental method (and which results in theory verification rather than disproof). A response is that these facts about the social psychology of scientific publication embed bias towards verification over disproof and limit the nature of theory building. In support of the alternative view of observational studies, one may note a recent trend to support replications of prior works. Further, in disciplines like medicine, it is increasingly seen as best practice to put research protocols to peer review prior to completing the experiments [33]. Some advocate presenting research proposals to the peer review system of scholarly journals [34]. Critiques of experimental studies include noting selective reporting of results, at odds with prior protocols [35]. This suggests that in some cases, it is the experimental research that is premature, and that research would benefit from prior scrutiny of both theory building associated with observational studies and the experimental protocol associated with experimental studies. The connection between the two is in being able to witness the efficacy of alternative operationalizations of theoretical constructs, and therefore enabling a wider and deeper understanding of how proposed experimental protocols might be varied.

Observational approaches are appropriate for establishing the ground truth for assessing emotional states and social behavioral changes in patients with dementia and their caregivers. The need of observational approaches in assistive technologies for dementia patients and their caregivers arises, as highlighted before, because these technologies should act as assistants and companions for patients, and assistants and knowledge repositories for their caregivers and healthcare professionals. In the context of patients with mental disorders, as dementia, it is expected that while interacting with the intelligent assistant and/or health care professionals, both patients and caregivers will express collections of emotional states and behavioral changes which hardly have been accounted by current artificial intelligence and machine learning approaches. The automatic detection of facets associated to such interactional exchanges requires endowing such intelligent devices of emotional and behavioral information very different from those aroused in categorical emotional contexts. In this situation, the involved emotions are far from the expected primary emotions of happiness, sadness, fear, disgust, surprise and anger [36].

Dyadic interactions between carers and dementia patients are prejudiced by different factors. As for caregivers, there will be latent emotional states of worry, weariness, anxiety, chronic stress, psychological illness and depressive symptoms related to carer's demographic factors, dementia characteristics of the carer's recipient, carer's burden, self-efficacy and received social support, and relationships (spouse, old child, etc.) between carer and carer's recipient. These emotional states may produce negative consequences not only on the carers' psychological and physical health but also on the quality of care they are able to release. These are the emotions that an observational approach would aim to identify to develop appropriate ICT devices/carers interactional models of assistance and support.

As for patients, neurodegenerative diseases are characterized by altered emotional responsiveness to salient sensory stimuli which mostly generate emotional dysregulation likely to be expressed through a wide spectrum of symptoms that 
impact the lives of patients and their caregivers. Such symptoms are abnormal responses to thermoregulatory and basic homeostatic signals [37], pathological seeking of rewarding stimuli such as food, sex or drugs [38], derangements of complex social behaviors [39] and emotional disturbances such as depression and anxiety [40]. We would expect to train intelligent devices to detect and recognize (from multimodal sources, mostly from speech and video-clips) these symptoms.

However, since these abilities require new models of behaviors there is a need to establish a ground truth for the proposed system evaluation. There is a need to foresee scenarios of human/human interactions to be later implemented for the human/agent interactions. These scenarios should elicit interactional exchanges among experimenters, patients, and caregivers. Data thusly collected need to be annotated by experts to provide the ground truth that will drive the assessment of the performances of these intelligent systems which in turn should learn from the data to detect early emotional and social behavioral changes in patients and caregivers. Only an observational approach can provide an initial set of labels for audio, video and biometric data segments collected during the interactions. These labels should account for caregivers' positive, negative and neutral emotional states as well as for stressed, worried, tired and depressive moods. Labels describing patients' behavioral changes (which may describe initial dementia symptoms) may include wandering, purposeless activity, fear of being alone, wounding, hurting, stealing, scratching, deviating, restlessness, rambling, agitation, aggressiveness, and restiveness to care [41]. They will pilot the annotation procedures and will be subjected to changes with the addition and/or deletion of labels as new knowledge and meaning will be uncovered while the observational approach is running and the collected data are analyzed.

\section{OBSERVING INTERACTION ANALYSIS PARADIGMS}

We have seen that the observational method is important in research that aspires to advance health care. Here we consider a moment of time in interaction analysis research, such as reported in the cognitive infocommunications conference series. We focus on the 10th IEEE international Conference on Cognitive Infocommunications, which took place in Naples, Italy during 2019. At this event, 110 research papers were presented. Of these, we deem 32 to be inside the line of research within cognitive infocommunications that is focused on linguistic and behavioural interaction analysis, including speech interaction: [1], [42]-[72].

Thus, far we have been contrasting observational and experimental methods. We have noted that both include hypothesis testing, but differ in whether the data collection controls contrasts from the outset or instead relevant contrasts available within data collected are explored. In examining the relevant works, it is evident that a range of other methods are also deployed and that few works use one method exclusively. However, thinking of the dominant approach within each, some natural clusters are evident: prospective, observational, experimental, technological, psychoanalytical, philosophical. Prospective works are those that have put forward for peer review a research protocol, for example, for an experiment or new technology. We think of a work as technological if the work involves evaluations of new technologies. We think of mathematical works as in the tradition of analytic philosophy, and deem them philosophical. Even considering dominance of methods, it nonetheless remains open that some works could be argued to fit more than one category (e.g. [61] which is largely technological, but also observational - the methods are experimental in evaluating technology, but using extant data sets, rather than constructing or harvesting a data-set for the purpose - below we classify this work as observational).

Our classifications follow: Prospective - [68], [71]; Observational - [45], [46], [50], [59], [61], [63], [64]; Experimental - [43], [58], [60]; Technological - [44], [49], [51]-[54], [56], [62], [67]; Psychoanalytical - [55], [65], [66], [69], [70], [72]; Philosophical - [1], [42], [47], [48], [57] 8] It may be observed that a range of methods are attested. Further, our own work embraces methodological plurality, as can be seen from authors of this paper contributing to works in more than one category - this paper is not proselytizing for observational research as the only true method. One might reasonably ask how we classify this paper: this section is observational and aspects are prospective, but the work as a whole is philosophical.

\section{CONCLUSION}

We think that the observational approach is ideal for studies closely linked to perception as a basis for annotation. The contrast between sentential syntax and the "grammar" of interaction is illuminating. There is little ambiguity when assigning a part of speech to a word. The annotators are based on their knowledge of grammar use, and validation of annotations can correct potential errors. However, when annotating laughter or dominance, annotators use their perception, which in turn is shaped by their knowledge of the world, their biases, their cultural background- factors that can be somehow controlled when recruiting annotators. Validation here mostly eliminates inconsistencies rather than accounting for errors. Perceptions are based on what we are exactly looking for: qualities and quantities that may or may not be linked to each other.

The MULTISIMO data set was not designed to determine the way, for example, collaboration or dominance would be manifested. We formulate hypotheses about how these concepts may be realized in a data-set like MULTISIMO, and we test whether relevant quantities interact with each other. An advantage is that new research questions may arise, even though the data-set hasn't been designed to address those questions. Given the challenges in constructing interaction data-sets, including recordings, ethics clearance, annotations etc., it appears to be best research practice to explore existing data for fresh observations, without limitation to a particular theoretical framework or a restricted number of observations.

We have seen that observational methods are well attested within interaction analysis and that the approach more or less defends itself 9 Therefore, we think it is not incumbent on researchers to defend the paradigm in their work, but rather demonstrate the appropriateness of an observational method (or, indeed, any other choice of methods) to their questions.

\footnotetext{
${ }^{8}$ We welcome debate regarding both the classification of these works and the appropriateness of including these, as opposed to others, as describing research in interaction analysis.

${ }^{9}$ If there is doubt about this, we hope that this paper may be relied upon as a defence of the observational paradigm.
} 


\section{ACKNOWLEDGMENT}

The research leading to these results has received funding from the ADAPT Centre (Grant 13/RC/2106), the European Regional Development Fund, the EU H2020 programme under the Marie Sklodowska-Curie grant No 701621.

\section{REFERENCES}

[1] C. Vogel and A. Esposito, "Linguistic and behavior interaction analysis within cognitive infocommunications," in 10th IEEE Conference on Cognitive Infocommunications, 2019, pp. 47-52.

[2] - "Interaction analysis and cognitive infocommunications," Infocommunications Journal, vol. 12, no. 1, pp. 2-9, 2020.

[3] M. T. Cicero, Rhetorica ad Herennium, ser. Loeb Classical Library. Harvard University Press, 95BC, 1954, Hary Caplan, (trans.); Authorship uncertain.

[4] M. F. Quintialianus, The Orator's Education, ser. Loeb Classical Library. Harvard University Press, 95CE, 2001, Donald Russell, (trans.).

[5] E. Goffman, Behavior in Public Places: Notes on the Social Organization of Gatherings. Free Press: New York, 1963.

[6] _- Interaction Ritual: Essays in Face-to-Face Behavior. New Brunswick, NJ: Transaction Publishers, 1967, reprinted 2008.

[7] A. L. Becker, "The linguistics of particularlity: Interpreting superordination in a Javanese text," in Beyond Translation: Essays toward a Modern Philology. Ann Arbor, MI: The University of Michigan Press, 1995, pp. 71-87, originally appeared in the Proceedings of the Tenth Annual Meeting of the Berkeley Linguistics Society, 1984.

[8] D. Biber, Variation across speech and writing. Cambridge University Press, 1988.

[9] M. Koutsombogera and C. Vogel, "Speech pause patterns in collaborative dialogs," in Innovations in Big Data Mining and Embedded Knowledge, ser. Intelligent Systems Reference Library, A. Esposito, A. M. Esposito, and L. C. Jain, Eds. Cham, Switzerland: Springer, 2019, pp. 99-115.

[10] G. R. Kuperberg and D. Caplan, "Language dysfunction in schizophrenia," in Neuropsychiatry, 2nd edition, R. Schiffer, S. Rao, and B. Fogel, Eds. Lippincott Williams and Wilkins, Philadelphia, PA, 2003, pp. 244-266.

[11] M. A. Covington, C. He, C. Brown, L. Naçi, J. T. McClain, B. S. Fjordbak, J. Semple, and J. Brown, "Schizophrenia and the structure of language: The linguists view," Schizophrenia Research, vol. 77, pp. 85-98, 2005.

[12] J. Cretchley, C. Gallois, H. Chenery, and A. Smith, "Conversations between carers and people with schizophrenia: A qualitative analysis using leximancer," Qualitative Health Research, vol. 20, no. 12, pp. 1611-1628, 2010. [Online]. Available: http://qhr.sagepub.com/content/ 20/12/1611.abstract

[13] G. R. Kuperberg, "Language in schizophrenia part 1: an introduction," Language \& Linguistics Compass, vol. 4, no. 8, pp. 576-589, 2010, doi:10.1111/j.1749-818X.2010.00216.x.

[14] A. Esposito, A. M. Esposito, and C. Vogel, "Needs and challenges in human computer interaction for processing social emotional information," Pattern Recognition Letters, vol. 66, pp. 41-51, 2015.

[15] J. C. S. J. Júnior, Y. Güçlütürk, M. Pérez, U. Güçlü, C. Andújar, X. Baró, H. J. Escalante, I. Guyon, M. A. J. van Gerven, R. van Lier, and S. Escalera, "First impressions: A survey on vision-based apparent personality trait analysis," 2018

[16] W. M. K. S. Ilmini and T. G. I. Fernando, "Computational personality traits assessment: A review," in 2017 IEEE International Conference on Industrial and Information Systems (ICIIS), 2017, pp. 1-6.

[17] A. Vinciarelli and G. Mohammadi, "A survey of personality computing," IEEE Transactions on Affective Computing, vol. 5, no. 3, pp. 273-291, 2014

[18] K. Luyckx and W. Daelemans, "Using syntactic features to predict author personality from text," in The 20th Joint International Conference of the Association for Literary and Linguistic Computing, and the Association for Computers and the Humanities (Digital Humanities 2008). The Association for Literary and Lingustic Computing, 2008, pp. 146-149.
[19] R. B. Long, "Paradigms for English verbs," Publications of the Modern Language Association, vol. 72, no. 3, pp. 359-372, 1957.

[20] T. S. Kuhn, The Structure of Scientific Revolutions. Chicago: University of Chicago Press, 1970.

[21] M. Masterman, "The nature of a paradigm," in Criticism and the Grown of Knowledge, I. Lakatos and A. Musgrave, Eds. Cambridge University Press, 1970, pp. 59-89.

[22] M. Koutsombogera and C. Vogel, "Observing collaboration in smallgroup interaction," Multimodal Technologies and Interaction, vol. 3 , no. 3, p. 45, 2019

[23] W. V. O. Quine, "Two dogmas of empiricism," in From a Logical Point of View. Cambridge, Mass: Harvard University Press, 1953, pp. 20-46.

[24] J. J. Godfrey, E. C. Holliman, and J. McDaniel, "Switchboard: telephone speech corpus for research and development," in Proceedings of ICASSP-92: 1992 IEEE International Conference on Acoustics, Speech, and Signal Processing, vol. 1, 1992, pp. 517-520.

[25] J. J. Godfrey and E. Holliman, "Switchboard-1 release 2," Philadephia, 1997, linguistic Data Consortium.

[26] A. H. Anderson, M. Bader, E. G. Bard, E. H. Boyle, G. M. Doherty, S. C. Garrod, S. D. Isard, J. C. Kowtko, J. M. McAllister, J. Miller, C. F. Sotillo, H. S. Thompson, and R. Weinert, "The herc map task corpus," Language and Speech, vol. 34, no. 4, pp. 351-366, 1992.

[27] L. Hunyadi, K. Bertók, T. E. Németh, I. Szekrényes, Á. Abuczki, G. Nagy, N. Nagy, P. Németi, and A. Bódog, "The outlines of a theory and technology of human-computer interaction as represented in the model of the hucomtech project," in 2011 2nd International Conference on Cognitive Infocommunications (CogInfoCom), 2011, pp. 1-5.

[28] L. Hunyadi, "On multimodality in the perception of emotions from materials of the hucomtech corpus," in 2015 6th IEEE International Conference on Cognitive Infocommunications (CogInfoCom), 2015, pp. 489-492.

[29] A. Hayakawa, S. Luz, L. Cerrato, and N. Campbell, "The ILMTs2s Corpus - A Multimodal Interlingual Map Task Corpus," in Proceedings of the Tenth International Conference on Language Reso urces and Evaluation (LREC 2016), N. C. C. Chair), K. Choukri, T. Declerck, M. Grobelnik, B. Maegaard, J. Mariani, A. Moreno, J. Odijk, and S. Piperidis, Eds. Paris, France: European Language Resources Association (ELRA), May 2016, pp. 605-612.

[30] M. Koutsombogera, R. Costello, and C. Vogel, "Quantifying dominance in the multisimo corpus," in 9th IEEE International Conference on Cognitive Infocommunications (CogInfoCom), P. Baranyi, A. Esposito, P. Földesi, and T. Mihálydeák, Eds., 2018, pp. 147-152.

[31] C. Vogel, M. Koutsombogera, and R. Costello, "Analyzing likert scale inter-annotator disagreement," in Neural Approaches to Dynamics of Signal Exchanges. Smart Innovation, Systems and Technologies, A. Esposito, M. Faundez-Zanuy, F. Morabito, and E. Pasero, Eds. Singapore: Springer, 2019, pp. 383-393.

[32] F. Eyben, M. Wöllmer, and B. Schuller, "OpenSMILE: The Munich Versatile and Fast Open-source Audio Feature Extractor," in Proceedings of the 18th ACM International Conference on Multimedia, ser. MM '10. New York, NY, USA: ACM, 2010, pp. 1459-1462.

[33] D. M. Yealy and K. H. Scruggs, "Study design and pre-trial, peer review in EMS research," Prehospital and Disaster Medicine, vol. 5, no. 2, pp. 113-117, 1990.

[34] G. Eysenbach, "Peer review and publication of research protocols and proposals: A role for open access journals," Journal of Medical Internet Research, vol. 6, no. 3, p. e37, 2004.

[35] A.-W. Chan, K. Krleža-Jerić, I. Schmid, and D. G. Altman, "Outcome reporting bias in randomized trials funded by the Canadian institutes of health research," vol. 171, no. 7, pp. 735-740, 2004

[36] P. Ekman, "An argument for basic emotions," Cognition and Emotion, vol. 6, no. 3-4, pp. 169-200, 1992. [Online]. Available: https://doi.org/10.1080/02699939208411068

[37] R. M. Ahmed, V. Iodice, N. Daveson, M. C. Kiernan, O. Piguet, and J. R. Hodges, "Autonomic dysregulation in frontotemporal dementia," Journal of Neurology, Neurosurgery \& Psychiatry, vol. 86, no. 9, pp. 1048-1049, 2015. [Online]. Available: https: //jnnp.bmj.com/content/86/9/1048

[38] D. Perry and J. Kramer, "Reward processing in neurodegenerative disease," Neurocase, vol. 21, no. 1, pp. 120-133, 2015. 
[39] P. Desmarais, K. L. Lanctt, M. Masellis, S. E. Black, and N. Herrmann, "Social inappropriateness in neurodegenerative disorders," International Psychogeriatrics, vol. 30, no. 2, pp. 197-207, 2018.

[40] V. Sturm, J. Yokoyama, W. Seeley, J. Kramer, B. Miller, and K. Rankin, "Heightened emotional contagion in mild cognitive impairment and alzheimer's disease is associated with temporal lobe degeneration," in Proceedings of the National Academy of Sciences of the United States of America, vol. 110, no. 24, 2013, pp. 9944-9949.

[41] Z. Ismail, L. Agera-Ortiz, H. Brodaty et al., "The mild behavioral impairment checklist (MBI-C): A rating scale for neuropsychiatric symptoms in pre-dementia populations," Journal of Alzheimer's Disease, vol. 56, no. 3, pp. 929-938, 2017.

[42] C. Vogel and A. Esposito, "Advancing and validating models of cognitive architecture," in 10th IEEE Conference on Cognitive Infocommunications, 2019, pp. 61-66.

[43] Z. G. Török and Á. Török, "Remember the north - : Reference frames and spatial cognition at different scale," in 2019 10th IEEE International Conference on Cognitive Infocommunications (CogInfoCom), 2019, pp. 21-26.

[44] N. Imre, R. Balogh, G. Gosztolya, L. Tóth, T. Várkonyi, C. Lengyel, M. Pákáski, and J. Kálmán, "Automatic recognition of temporal speech features in type 2 diabetes mellitus with mild cognitive impairment," in 2019 10th IEEE International Conference on Cognitive Infocommunications (CogInfoCom), 2019, pp. 27-28.

[45] A. Esposito, T. Amorese, M. Buonanno, M. Cuciniello, A. M. Esposito, M. Faundez-Zanuy, L. Likforman-Sulem, M. T. Riviello, A. Troncone, and G. Cordasco, "Handwriting and drawing features for detecting personality traits," in 2019 10th IEEE International Conference on Cognitive Infocommunications (CogInfoCom), 2019, pp. 79-84.

[46] B. K. Szabó, "Interaction in an immersive virtual reality application," in 2019 10th IEEE International Conference on Cognitive Infocommunications (CogInfoCom), 2019, pp. 35-40.

[47] P. B. Graben, R. Römer, W. Meyer, M. Huber, and M. Wolff, "Reinforcement learning of minimalist numeral grammars," in 2019 10th IEEE International Conference on Cognitive Infocommunications (CogInfoCom), 2019, pp. 67-72.

[48] R. Römer, P. B. Graben, M. Huber, W. Matthias, G. Wirsching, and I. Schmitt, "Behavioral control of cognitive agents using database semantics and minimalist grammars," in 2019 10th IEEE International Conference on Cognitive Infocommunications (CogInfoCom), 2019, pp. 73-78.

[49] A. Ito and K. Nakada, "Ui design based on traditional japanese gesture," in 2019 10th IEEE International Conference on Cognitive Infocommunications (CogInfoCom), 2019, pp. 85-90.

[50] B. Berki, "Sense of presence in maxwhere virtual reality," in 2019 10th IEEE International Conference on Cognitive Infocommunications (CogInfoCom), 2019, pp. 91-94.

[51] D. Hládek, J. Staš, and S. Ondáš, "Comparison of recurrent neural networks for slovak punctuation restoration," in 2019 10th IEEE International Conference on Cognitive Infocommunications (CogInfoCom), 2019, pp. 95-100.

[52] R. Darshi, A. R. Khayatian, M. Dehghani, and R. Boostani, "Modelling and control of paraplegic subjects walking using functional electrical stimulation," in 2019 10th IEEE International Conference on Cognitive Infocommunications (CogInfoCom), 2019, pp. 113-118.

[53] S. Ondáš, D. Hládek, M. Pleva, J. Juhár, E. Kiktová, J. Zimmermann, and M. Oravcová, "Towards robot-assisted children speech audiometry," in 2019 10th IEEE International Conference on Cognitive Infocommunications (CogInfoCom), 2019, pp. 119-124.

[54] B. Tarján, G. Szaszák, T. Fegyó, and P. Mihajlik, "N-gram approximation of 1stm recurrent language models for single-pass recognition of hungarian call center conversations," in 2019 10th IEEE International Conference on Cognitive Infocommunications (CogInfoCom), 2019, pp. 131-136.

[55] V. Bochicchio, N. M. Maldonato, R. Vitelli, and C. Scandurra, “"emotional nose": The hedonic character of olfaction and its epistemological and clinical implications," in 2019 10th IEEE International Conference on Cognitive Infocommunications (CogInfoCom), 2019, pp. 143-146.

[56] M. Koctúrová and J. Juhár, "Speech activity detection from eeg using a feed-forward neural network," in 2019 10th IEEE International
Conference on Cognitive Infocommunications (CogInfoCom), 2019, pp. 147-152.

[57] P. Klimczak, K. Nowack, and G. Wirsching, "A quantum cognition model for learning by difference," in 2019 10th IEEE International Conference on Cognitive Infocommunications (CogInfoCom), 2019, pp. 163-168.

[58] L. Oestreicher, "Cognitive chunks as neural activity : Is it possible to see what you think?" in 2019 10th IEEE International Conference on Cognitive Infocommunications (CogInfoCom), 2019, pp. 199-204.

[59] C. Navarretta, "Form and function of hand gestures for interpretation and generation," in 2019 10th IEEE International Conference on Cognitive Infocommunications (CogInfoCom), 2019, pp. 215-220.

[60] A. Karampela and C. Vogel, "Nouns and verbs in professional reporting of extreme events," in 2019 10th IEEE International Conference on Cognitive Infocommunications (CogInfoCom), 2019, pp. 253-258.

[61] G. Szaszak and P. Pierucci, "A comparative analysis of domain adaptation techniques for recognition of accented speech," in 2019 10th IEEE International Conference on Cognitive Infocommunications (CogInfoCom), 2019, pp. 259-264.

[62] M. d. Vázquez, R. Justo, A. L. Zorrilla, and M. I. Torres, "Can spontaneous emotions be detected from speech on TV political debates?" in 2019 10th IEEE International Conference on Cognitive Infocommunications (CogInfoCom), 2019, pp. 289-294.

[63] D. Sztahó and I. Valálik, "Speech fluency measurement of patients with parkinsons disease by forward-backward divergence segmentation," in 2019 10th IEEE International Conference on Cognitive Infocommunications (CogInfoCom), 2019, pp. 295-300.

[64] M. G. Tulics, G. Szaszák, K. Mészáros, and K. Vicsi, "Artificial neural network and svm based voice disorder classification," in 2019 10th IEEE International Conference on Cognitive Infocommunications (CogInfoCom), 2019, pp. 307-312.

[65] N. M. Maldonato, M. Bottone, R. Sperandeo, C. Scandurra, V. Bochicchio, M. L. Cappuccio, A. M. Esposito, and B. Muzii, "The biodynamic stress hypothesis towards an evolutionary psychology paradigm," in 2019 10th IEEE International Conference on Cognitive Infocommunications (CogInfoCom), 2019, pp. 369-374.

[66] N. M. Maldonato, B. Muzii, D. Di Corrado, R. Sperandeo, M. Bottone, M. L. Cappuccio, M. Coco, and A. Esposito, "The spontaneous order of creativity brain, complexity and evolution," in 2019 10th IEEE International Conference on Cognitive Infocommunications (CogInfoCom), 2019, pp. 375-380.

[67] V. Szücs, T. Guzsvinecz, and A. Magyar, "Improved algorithms for movement pattern recognition and classification in physical rehabilitation," in 2019 10th IEEE International Conference on Cognitive Infocommunications (CogInfoCom), 2019, pp. 417-424.

[68] B. Tolgay, S. DellOrco, N. M. Maldonato, C. Vogel, L. Trojano, and A. Esposito, "Eegs as potential predictors of virtual agents acceptance," in 2019 10th IEEE International Conference on Cognitive Infocommunications (CogInfoCom), 2019, pp. 433-438.

[69] R. Sperandeo, L. L. Mosca, Y. M. Alfano, V. Cioffi, A. D. Di Sarno, A. Galchenko, D. Iennaco, T. Longobardi, E. Moretto, S. DellOrco, B. Muzii, and N. M. Maldonato, "Complexity in the narration of the self a new theoretical and methodological perspective of diagnosis in psychopathology based on the computational method," in 2019 10th IEEE International Conference on Cognitive Infocommunications (CogInfoCom), 2019, pp. 445-450.

[70] M. Coco, A. Buscemi, C. S. Guerrera, C. Licitra, E. Pennisi, V. Vettor, L. Rizzi, P. Bovo, P. Fecarotta, S. DellOrco, B. Muzii, M. Bottone, M. Bellomo, and D. Di Corrado, "Touch and communication in the institutionalized elderly," in 2019 10th IEEE International Conference on Cognitive Infocommunications (CogInfoCom), 2019, pp. 451-458.

[71] R. Sperandeo, L. L. Mosca, Y. M. Alfano, V. Cioffi, A. D. Di Sarno, A. Galchenko, D. Iennaco, T. Longobardi, E. Moretto, S. DellOrco, B. Muzii, and N. M. Maldonato, "Preventing dyspraxia: a project for the creation of a computational diagnostic system based on the theory of embodied cognition," in 2019 10th IEEE International Conference on Cognitive Infocommunications (CogInfoCom), 2019, pp. 511-512.

[72] D. Nergui-Szöllössy, "Object classification as key for algorithmic language processing of metonymy and metaphor : A sensory language retrieval approach+," in 2019 10th IEEE International Conference on Cognitive Infocommunications (CogInfoCom), 2019, pp. 605-614. 